\section{Prevalência e fatores associados ao uso de antiinflamatórios não- esteróides por pacientes submetidos a endoscopia digestiva alta, Belo Horizonte, Minas Gerais, 2000}

\section{Prevalence of and factors associated with nonsteroidal anti- inflammatory drug use by patients submitted to upper endoscopy, Belo Horizonte, Minas Gerais, 2000}

\section{Andréia Queiroz Ribeiro'*}

\section{Gil Sevalho'}

\section{Cibele Comini César ${ }^{2}$}

'Departamento de Farmácia Social, Faculdade de Farmácia, Universidade Federal de Minas Gerais

${ }^{2}$ Instituto de Ciências Exatas, Universidade Federal de Minas Gerais

\section{Resumo}

Objetivo: Determinar a prevalência e os fatores associados ao uso de AINE por pacientes submetidos a endoscopia digestiva alta no Hospital das Clínicas da UFMG. Métodos: Estudo transversal de uma amostra de 533 pacientes com idade igual ou superior a 17 anos, com endoscopia previamente marcada na Seção de Endoscopia do Hospital das Clínicas da Universidade Federal de Minas Gerais. Os dados foram coletados por meio de entrevistas. Foram considerados quatro grupos de variáveis exploratórias: sociodemográficas, relacionadas aos hábitos de vida, relacionadas à história de morbidades e relacionadas ao uso de medicamentos. Os dados foram submetidos às análises estatísticas bivariada e multivariada. Resultados e Conclusões: Entre os entrevistados, $34,1 \%$ relataram algum uso de AINE no período de 1 mês anterior à realização da endoscopia. Os AINE mais utilizados foram o ácido acetilsalicílico e o diclofenaco. Os fatores associados ao uso de AINE foram: sexo feminino $(\mathrm{OR}=2,07$; IC $95 \%=1,28$ $3,34)$, renda igual ou superior a 3 salários mínimos (OR =3,20; IC 95\% = 1,74-5,90), uso de álcool $(\mathrm{OR}=2,43$; IC 95\% = 1,39-4,24), presença de sintomas gastrintestinais $(\mathrm{OR}=$ 1,82; IC $95 \%=1,18-2,80$ ), uso regular de 4 ou mais medicamentos $(\mathrm{OR}=4,33$; IC $95 \%=$ 2,49-7,54) e história prévia de úlcera e/ou hemorragia digestiva $(\mathrm{OR}=0,40$; IC $95 \%=$ $0,22-0,75)$. Estes resultados mostram semelhanças aos observados em países desenvolvidos. Além disso, alertam para a necessidade de maior atenção por profissionais de saúde para com os subgrupos de uso evidenciados.

Palavras-chave: Farmacoepidemiologia. Prevalência. Antiinflamatórios não-esteróides. Endoscopia digestiva alta. 


\section{Abstract}

Introduction: The objective was to determine the prevalence and the factors associated with nonsteroidal anti-inflammatory drugs (NSAID) used by patients submitted to upper endoscopy at Hospital das Clínicas / UFMG, Belo Horizonte, MG. Methods: Cross-sectional study of a 533 patients, aged 17 or older, whose endoscopies had been previously scheduled at the Endoscopy Section of Hospital das Clinicas, of the Federal University of Minas Gerais. Data were collected in interviews. Study variables were divided into four groups: social and economic, life habits, morbidity, and drug use. Data were submitted to bivariate and multivariate statistical analyses. Results and Conclusions: A percentage of $34.1 \%$ of the interviewed sample reported the use of NSAID drugs in the period of one month before the endoscopy. The most frequently used NSAID were acetylsalicylic acid and diclofenac. The factors associated with NSAID use were: female gender $(\mathrm{OR}=2.07$; CI 95\% = 1.28-3.34), monthly income 33 minimum-wages $(\mathrm{OR}=$ 3.20; CI 95\% = 1.74-5.90), alcohol use (OR = $2.43 ;$ CI $95 \%=1.39-4.24)$, gastric symptoms $(\mathrm{OR}=1.82$; CI 95\% = 1.18-2.80), regular use of 4 or more drugs $(\mathrm{OR}=4.33$; CI 95\% $=2.49$ 7.54) and previous history of peptic ulcer and/or digestive hemorrhage $(\mathrm{OR}=0.40$; $\mathrm{CI}$ $95 \%=0.22-0.75)$. These results are similar to others from developed countries. Moreover, they point towards to the need of greater care by health professionals for the subgroups identified in this study.

Keywords: Pharmacoepidemiology. Prevalence. Nonsteroidal anti-inflammatory agents. Upper endoscopy.

\section{Introdução}

Os antiinflamatórios não-esteróides (AINE) integram o grupo dos fármacos mais comumente prescritos em todo o mundo e estão entre os mais utilizados nas práticas de automedicação ${ }^{1-4}$. Nos Estados Unidos, respondem por mais de 70 milhões de prescrições e mais de 30 bilhões de comprimidos de venda livre comercializados anualmente $^{5}$. No Brasil, diferentes estudos de utilização de medicamentos situam os AINE entre os mais utilizados pela população ${ }^{6-8}$.

No âmbito internacional, muitos estudos discutem a importância de fatores como sexo e idade na determinação do uso de AINE. Neste sentido, mostram que os AINE são mais comumente utilizados pelas mulheres, enquanto a idade não é um forte preditor da utilização dos mesmos ${ }^{9-11}$. Outros fatores, tais como hábitos de vida e presença de morbidades específicas, sobretudo relacionadas à dor, também têm sido discutidos como possíveis determinantes do uso destes fármacos ${ }^{2,9,12}$.

No Brasil, estudos específicos sobre a utilização destes fármacos, bem como sobre os fatores relacionados a este uso, são raros ou mesmo inexistentes, embora o conhecimento destas questões seja imprescindível para o emprego terapêutico adequado desta classe.

O presente trabalho refere-se a um estudo epidemiológico sobre a prevalência e os fatores associados ao uso de AINE por pacientes submetidos à endoscopia digestiva alta. O estudo foi desenvolvido com os seguintes objetivos: determinar a prevalência do uso deAINE entre indivíduos com 17 ou mais anos de idade; identificar os principais representantes desta classe utilizados por essa população; e determinar os fatores sociodemográficos, relacionados aos hábitos de vida, relacionados à história de morbidades e ao uso concomitante de outros medicamentos independentemente associados ao uso de AINE.

\section{Métodos}

O presente estudo integra uma pesquisa 
sobre o uso de AINE e a ocorrência de lesões do trato gastrintestinal em pacientes submetidos a endoscopia digestiva alta (EDA) no Hospital das Clínicas da UFMG (HC/UFMG), realizada no ano de 2000 . A pesquisa foi aprovada pelo Comitê de Ética em pesquisa do referido hospital, de acordo com o parecer de número ETIC 007/00 de 24 de abril de 2000.

A população de referência corresponde aos indivíduos com 17 anos ou mais de idade, com EDA previamente marcada. Foram excluídos pacientes sem condição de resposta e pacientes submetidos a procedimentos endoscópicos específicos, tais como escleroterapia de varizes esofagianas, ligadura elástica de varizes, dilatação de estenose do esôfago ou de obstrução pilórica e polipectomia. Para o cálculo da amostra adotouse uma prevalência do evento de interesse igual a 0,5, nível de confiança igual a 95\% e a margem de erro tolerável de 0,05 , o que resultou em 385 pacientes. Considerando uma perda de 15\%, um tamanho amostral de 445 pacientes seria suficiente. Entretanto, a amostra incluiu 533 pacientes, os quais foram selecionados por amostragem sistemática. Detalhes sobre o processo de amostragem encontram-se em Ribeiro ${ }^{13}$.As informações foram obtidas por entrevista realizada anteriormente à realização do exame endoscópico, entre junho e dezembro de 2000, utilizando-se um questionário padronizado.

\section{Variáveis do estudo}

A variável resposta foi o uso de AINE no período de 30 dias anteriores à realização da endoscopia. Foram coletadas informações sobre qualquer tipo de uso, contínuo ou não, no período estudado. Não se considerou, nesta abordagem, o uso de dipirona e paracetamol, uma vez que esses não se relacionam a lesões do trato gastrintestinal. A partir desta informação, os pacientes foram classificados em dois grupos: o primeiro grupo compreende os pacientes que relataram qualquer uso de AINE no período considerado e o segundo grupo refere-se àqueles que não relataram qualquer uso destes fármacos no mesmo período.

Foram considerados quatro grupos de covariáveis: sociodemográficas, relacionadas aos hábitos de vida, relacionadas à história de morbidades e relacionadas ao uso de medicamentos (exceto AINE).

As variáveis sociodemográficas foram as seguintes: sexo, idade, escolaridade (anos) e renda individual mensal em salários-mínimos da época.

As variáveis relacionadas aos hábitos de vida foram: uso de álcool (você bebe alguma bebida alcoólica?) e uso de cigarro (você fuma?).

As variáveis referentes à história de morbidades compreendem: realização da endoscopia pela primeira vez, motivo da endoscopia (sintomas gastrintestinais - dor, cólica, pirose, queimação - ou outro motivo) e história prévia de úlcera e/ou hemorragia digestiva.

As variáveis sobre uso de medicamentos que não AINE foram: o número de medicamentos utilizados regularmente e o uso de corticosteróides (nos últimos 30 dias).

\section{Análise dos dados}

Para a análise bivariada dos dados empregou-se o teste do qui-quadrado de Pearson e o teste exato de Fisher. A associação independente entre as variáveis e o uso de AINE foi verificada na análise multivariada utilizando-se a regressão logística. O critério para inclusão de variáveis no modelo logístico foi a associação com o uso de AINE em nível de $\mathrm{p}<0,20$ na análise bivariada. No entanto, as variáveis uso de álcool e uso de cigarro foram incluídas nos modelos, pois as consideramos importantes do ponto de vista epidemiológico. O critério de permanência das variáveis no modelo foi a associação com o uso de AINE em nível de p<0,05. A medida de associação selecionada foi a razão de chances (odds ratio), que é apresentada como odds ratio bruto (quando não houve controle por fatores de confusão) e odds ratio ajustado (a medida de associação resultante da análise, controlando-se por 
fatores de confusão). A análise dos dados foi feita utilizando-se o software Stata (Versão 7.0)

\section{Resultados}

Do total de 533 indivíduos estudados, 182 $(34,1 \%)$ relataram uso de algum AINE durante algum período nos 30 dias que antecederam a entrevista, totalizando 249 medicamentos utilizados. Dentre esses, a maioria (70,9\%) utilizou apenas um AINE no período estudado. Entretanto, 6,6\% dos usuários relataram o uso de três ou mais antiinflamatórios nãoesteróides no período considerado.

A principal característica foi de uso esporádico e por período inferior a sete dias $(51,4 \%)$. Por outro lado, entre os pacientes que relataram uso contínuo de AINE, a maioria o fez por um período superior a 30 dias. Continuamente ou de maneira esporádica, 25,7\% dos AINE foram utilizados por mais de 30 dias. $\mathrm{O}$ fármaco mais utilizado foi o ácido acetilsalicílico (59,5\%), seguido do diclofenaco $(28,1 \%)$ e do piroxicam $(2,8 \%)$.

A Tabela 1 mostra a distribuição do uso de AINE de acordo com as variáveis sociodemográficas selecionadas. Sexo e renda indi- vidual mostraram-se significativamente associados ao uso de AINE $(\mathrm{p}<0,05)$. A idade foi associada de forma positiva, mas com uma significância limítrofe.

De acordo com a Tabela 2, pode-se observar que não houve associação estatisticamente significativa entre o uso de álcool e o uso de cigarro com a utilização de AINE.

Em relação aos indicadores de história de morbidades, observa-se na Tabela 3 que a realização de endoscopia pela primeira vez, sintomas gastrintestinais como motivo da endoscopia e história prévia de úlcera e/ou hemorragia digestiva associaram-se de forma significante com o uso de AINE $(p<0,05)$.

Na Tabela 4 está apresentada a distribuição do uso de AINE segundo o número de medicamentos (que não AINE) usados regularmente e o uso de corticosteróide. Observa-se que o uso regular de quatro ou mais medicamentos e o uso de corticosteróide associam-se de forma significativa ao uso de AINE $(\mathrm{p}<0,05)$.

Na Tabela 5 estão apresentados os resultados finais da análise multivariada dos fatores associados ao uso de AINE. As seguintes variáveis apresentaram associações independentes com o uso de AINE: sexo femini-

Tabela 1 - Distribuição do uso de AINE de acordo com variáveis sociodemográficas, Belo Horizonte (MG), 2000. $N=533$

Table 1 - Distribution of NSAID use according to socio-demographic variables, Belo Horizonte (MG), 2000. $N=533$

\begin{tabular}{|c|c|c|c|}
\hline \multirow[t]{2}{*}{ Variáveis sóciodemográficas } & \multicolumn{2}{|c|}{ Uso de AINE } & \multirow[t]{2}{*}{ Valor $\mathrm{p}$} \\
\hline & não & $\operatorname{sim}$ & \\
\hline \multicolumn{4}{|l|}{ Sexo } \\
\hline masculino (\%) & $153(71,2)$ & $62(28,8)$ & \\
\hline Feminino (\%) & $198(62,3)$ & $120(37,7)$ & $p=0,03$ \\
\hline \multicolumn{4}{|l|}{ Idade } \\
\hline$<60$ anos (\%) & $255(68,6)$ & $117(31,4)$ & \\
\hline$\geq 60$ anos $(\%)$ & $96(59,6)$ & $65(40,4)$ & $p=0,05$ \\
\hline \multicolumn{4}{|l|}{ Escolaridade } \\
\hline 0 ano de estudo (\%) & & $39(58,2)$ & $28(41,8)$ \\
\hline 1 ano ou mais de estudo (\%) & $312(67,0)$ & $154(33,0)$ & $p=0,16$ \\
\hline \multicolumn{4}{|l|}{ Renda individual (SM)* } \\
\hline < 3 SM (\%) & $290(67,9)$ & $137(32,1)$ & \\
\hline$\geq 3$ SM (\%) & $61(57,6)$ & $45(42,4)$ & $p=0,04$ \\
\hline
\end{tabular}

*SM: salário-mínimo à época do estudo: $\mathrm{R} \$ 151,00$

* SM: minimum wage during the period of study: US\$82.95 
Tabela 2 - Distribuição do uso de AINE de acordo com variáveis relacionadas aos hábitos de vida, Belo Horizonte (MG), 2000. N= 533

Table 2 - Distribution of NSAID use according to lifestyle variables, Belo Horizonte (MG), 2000. N= 533

\begin{tabular}{lccc}
\hline Variáveis relacionadas aos hábitos de vida & \multicolumn{2}{c}{ Uso de AINE } & Valor p \\
& não $(\%)$ & $\operatorname{sim}(\%)$ & \\
\hline Uso de álcool & & & \\
$\quad$ não & $230(67,2)$ & $112(32,8)$ & \\
$\quad$ sim & $121(63,3)$ & $70(36,7)$ & $\mathrm{p}=0,362$ \\
Uso de cigarro & & & \\
$\quad$ não & $286(65,9)$ & $148(34,1)$ & \\
$\quad$ sim & $65(65,7)$ & $34(34,3)$ & $\mathrm{p}=0,963$ \\
\hline
\end{tabular}

Tabela 3 - Distribuição do uso de AINE de acordo com as variáveis relacionadas à história de morbidades, Belo Horizonte (MG), 2000. $\mathrm{N}=533$

Table 3 -Distribution of NSAID use according to morbidity variables, Belo Horizonte (MG), 2000. N= 533

\begin{tabular}{lccc}
\hline Variáveis de história de morbidades & \multicolumn{2}{c}{ Uso de AINE } & Valor p \\
& não & $\operatorname{sim}$ & \\
\hline $\begin{array}{l}\text { Primeira endoscopia } \\
\text { não (\%) }\end{array}$ & $223(70,6)$ & $93(29,4)$ & \\
$\quad$ sim (\%) & $128(59,0)$ & $89(41,0)$ & $\mathrm{p}<0,01$ \\
Motivo da endoscopia* & & & \\
$\quad$ outro motivo (\%) & $165(75,7)$ & $53(24,3)$ & $\mathrm{p}<0,01$ \\
$\quad$ sintomas gastrintestinais (\%) & $184(59,3)$ & $126(40,7)$ & \\
História prévia de úlcera/ hemorragia** & & & \\
$\quad$ não (\%) & $255(61,6)$ & $159(38,4)$ & $\mathrm{p}<0,01$ \\
$\quad$ sim (\%) & $86(84,3)$ & $16(15,7)$ &
\end{tabular}

${ }^{*} \mathrm{~N}=528 ;{ }^{* *} \mathrm{~N}=516$

Tabela 4 - Distribuição do uso de AINE de acordo com as variáveis relacionadas ao uso de medicamentos, Belo Horizonte (MG), 2000. N = 533

Table 4-Distribution of NSAID use according to variables related to drug use, Belo Horizonte (MG), 2000. $N=533$

\begin{tabular}{|c|c|c|c|}
\hline \multirow{2}{*}{$\begin{array}{l}\text { Variáveis relacionadas ao uso de outros } \\
\text { medicamentos }\end{array}$} & \multicolumn{2}{|c|}{ Uso de AINE } & \multirow[t]{2}{*}{ Valor $p$} \\
\hline & não & $\operatorname{sim}$ & \\
\hline \multicolumn{4}{|l|}{$\mathrm{N}^{\circ}$ de medicamentos usados regularmente } \\
\hline 1 - 3 medicamentos (\%) & $319(72,2)$ & $123(27,8)$ & \\
\hline$\geq 4$ medicamentos $(\%)$ & $32(35,2)$ & $59(64,8)$ & $\mathrm{p}<0,01$ \\
\hline \multicolumn{4}{|l|}{ Uso de corticosteróide } \\
\hline não (\%) & $347(67,1)$ & $170(32,9)$ & \\
\hline $\operatorname{sim}(\%)$ & $4(25,0)$ & $12(75,0)$ & $\mathrm{p}<0,01^{*}$ \\
\hline
\end{tabular}

* teste exato de Fisher 
Tabela 5 - Resultados finais da análise multivariada dos fatores associados ao uso de AINE, Belo Horizonte (MG), 2000. $\mathrm{N}=517$

Table 5 - Final results of the multivariate analysis of factors associated with NSAID use, Belo Horizonte (MG), 2000. $N=517$

\begin{tabular}{|c|c|c|c|c|}
\hline \multirow[t]{2}{*}{ Variáveis } & \multicolumn{2}{|c|}{ Bruto } & \multicolumn{2}{|c|}{ Ajustado } \\
\hline & OR & IC $95 \%$ & OR & IC 95\% \\
\hline Sexo feminino & 1,50 & $1,01-2,22$ & 2,07 & $1,28-3,34$ \\
\hline Anos de escolaridade & 0,98 & $0,95-1,03$ & 0,91 & $0,86-0,96$ \\
\hline Renda $\geq 3 \mathrm{SM}$ & 1,56 & $0,98-2,48$ & 3,20 & $1,74-5,90$ \\
\hline Uso de álcool & 1,18 & $0,82-1,72$ & 2,43 & $1,39-4,24$ \\
\hline $\begin{array}{l}\text { Sintomas gastrintestinais como } \\
\text { motivo da endoscopia }\end{array}$ & 2,13 & $1,42-3,20$ & 1,82 & $1,18-2,80$ \\
\hline $\begin{array}{l}\text { História prévia de úlcera e/ou } \\
\text { hemorragia }\end{array}$ & 0,30 & $0,16-0,55$ & 0,40 & $0,22-0,75$ \\
\hline $\begin{array}{l}\text { Uso regular de quatro ou } \\
\text { mais medicamentos }\end{array}$ & 4,78 & $2,88-7,97$ & 4,33 & $2,49-7,54$ \\
\hline
\end{tabular}

no $(\mathrm{OR}=2,07$; IC95\%=1,28 - 3,34); anos de escolaridade $(\mathrm{OR}=0,91 ; \mathrm{IC} 95 \%=0,86-0,96)$; renda igual ou superior a três salários-mínimos $(\mathrm{OR}=3,20$; IC95\%= $1,74-5,90)$; uso de bebida alcoólica $(\mathrm{OR}=2,43$; IC95\%=1,394,24); presença de sintomas gastrintestinais como motivo da endoscopia $(\mathrm{OR}=1,82$; IC95\%=1,18-2,80); história prévia de úlcera e/ou hemorragia digestiva $(\mathrm{OR}=0,4$; IC95\%=0,22-0,75); e uso regular de quatro ou mais medicamentos $(\mathrm{OR}=4,33$; IC95\% $=2,49$ $7,54)$. A força da associação entre sexo feminino e uso de AINE aumentou após o controle por renda e uso de álcool. Para a associação entre escolaridade e uso de AINE, a renda foi o principal confundidor. No caso da renda, os principais fatores de confusão foram sexo, escolaridade e número de medicamentos usados. Enfim, para o uso de álcool,o principal fator de confusão foi o número de medicamentos utilizados.

\section{Discussão}

A prevalência do uso de AINE entre pacientes submetidos à EDA no $\mathrm{HC}$ foi de $34,1 \%$ no período investigado. A posição de destaque do AAS pode se dever tanto ao fato de ele ser, entre os diferentes AINE, o principal componente de combinações antiácidas e antigripais, bem como por seu uso difundi- do como antiagregante plaquetário. Em que pesem as diferenças metodológicas, nossos resultados se assemelham aos de outros estudos realizados na Finlândia e no Rio de Janeiro $^{9,14}$. Por outro lado, são diferentes do encontrado na Itália, em especial no que concerne aos principais fármacos utilizados, quando se destacaram, naquele país, a nimesulida, o ácido acetilsalicílico e o ibuprofeno ${ }^{1}$.

$\mathrm{O}$ uso de AINE foi mais freqüente entre as mulheres, ocasionando uma associação positiva e significativa entre o sexo feminino e o uso de AINE. Esta tendência é confirmada na grande maioria dos estudos, sejam eles baseados em dados de prescrição ou ainda aqueles que consideram o uso de AINE não prescritos ${ }^{1,2,4,9-11,14-16}$. Uma vez que várias são as razões para este perfil, o maior interesse nesta questão refere-se à maior propensão das mulheres para receberem prescrições medicamentosas e para se automedicarem, fato este que as tornam mais vulneráveis aos prejuízos oriundos do uso de medicamentos, como um maior risco de reações adversas. Esta situação orienta para uma maior atenção dos prescritores para com as mulheres, uma vez que estas se encontram mais expostas ao uso de múltiplos fármacos e, conseqüentemente, aos efeitos prejudiciais advindos desta prática. 
A idade é motivo de controvérsias ao predizer o uso de AINE em diferentes estudos. É coerente pensar-se que o uso de AINE aumenta na medida em que a idade avança, tendo em vista o aumento da freqüência de doenças crônicas, muitas das quais requerem o uso desta classe de medicamentos. No entanto, um importante fator interveniente nesta relação é a fonte de indicação deste uso: prescrição ou não. Nesta perspectiva, a maioria dos estudos que demonstra uma associação positiva e constante com a idade e o uso de AINE baseia-se unicamente em registros de prescrições médicas $^{10,17,18}$, ao passo que os estudos que incluem também o uso de AINE não prescritos, não evidenciam este relacionamento $0^{9,11,16} . \mathrm{Na}$ análise bivariada, a associação entre a idade igual ou superior a 60 anos e o uso de AINE foi positiva; entretanto, esta não se manteve na análise multivariada, após controle por fatores de confusão. Esta ausência de associação pode se dever ao fato de o estudo contemplar o uso de AINE não prescrito e este, além de ter sido o tipo mais freqüente de uso, ter sido praticado, em sua maioria, por adultos jovens. De acordo com alguns autores, o uso de medicamentos não prescritos é mais comum em adultos jovens, enquanto o uso de prescritos ocorre com maior freqüência entre idosos ${ }^{11}$, e esta situação também ocorre em relação aos AINE. Embora referentes a uma população específica, nossos resultados acompanharam esta tendência.

Ainda no que diz respeito às características sociodemográficas, alguns poucos estudos sugerem que a condição social e o nível educacional são de menor importância como determinantes do uso de AINE/analgésicos ${ }^{9,11}$, ao passo que outros evidenciam uma associação entre melhor condição social e uso de $\mathrm{AINE}^{2}$. No presente estudo, a maior renda associou-se ao uso destes medicamentos e a maior escolaridade associouse negativamente, embora nesta última o intervalo de confiança tenha sido limítrofe, com o limite superior situado próximo à unidade.

São escassos os estudos que enfocam o relacionamento entre hábitos de vida e o uso de AINE. Dentre os existentes, alguns apontam o cigarro e o álcool como importantes determinantes ${ }^{9,11,12}$, enquanto outros os apresentam com um fraco relacionamento com o uso dos referidos fármacos ${ }^{2}$. Uma razão possível para o primeiro achado é que os indivíduos que utilizam substâncias como cigarro e álcool sejam mais vulneráveis a problemas de saúde e consumam com maior freqüência substâncias como os analgésicos, podendo-se inserir os AINE nessa condição $0^{9,11,12}$. No estudo de Ferguson $(1973)^{12} \mathrm{e}$ no estudo de Antonov \& Isacson (1996) ${ }^{9}$, ambos os usos, de cigarro e álcool, se relacionaram ao uso de AINE/analgésicos. No nosso estudo, somente o uso de álcool foi associado positivamente com o uso dos fármacos em questão.

Considerando as características da população estudada, os resultados do presente estudo são mais preocupantes por se referirem a indivíduos provenientes, em sua maioria, de clínicas de gastroenterologia e, por conseguinte, já apresentarem alguma história de morbidades digestivas. Em função disso, eles estão expostos a um maior risco de ocorrência de lesões e complicações gastrintestinais, que, na presença do uso de AINE, pode se tornar ainda maior, apesar das controvérsias. Vale ainda ressaltar que, por se tratar de um estudo exploratório, não quantificamos o uso de álcool e cigarro, o que pode influenciar diferentemente o uso de AINE. Estudos posteriores são necessários para uma melhor compreensão desta relação.

Vários estudos são unânimes no que diz respeito ao fato da dor e das doenças a ela relacionadas serem consideradas como os "motivadores legítimos" para o uso de analgésicos ("painkillers"). Neste âmbito podese incluir os antiinflamatórios não-esteroidais, uma vez que além da ausência de clara distinção, por parte da população, entre analgésicos e antiinflamatórios, o maior uso destes ocorre com finalidade analgési$\mathrm{ca}^{1,9,11,16,17}$. Paradoxalmente, a literatura também aponta que o fator mais limitante na adesão dos pacientes ao tratamento com 
AINE são as reações adversas gastrintestinais, principalmente a dispepsia ${ }^{17}$. Na nossa investigação, apesar da associação encontrada entre a presença de sintomas gastrintestinais e o uso de AINE poder ser atribuída, em parte, à alta freqüência do uso de combinações contendo AINE com finalidade antiácida, uma limitação inerente ao desenho transversal é a impossibilidade de se estabelecer com certeza a direção desta associação. É difícil distinguir quando os sintomas gastrintestinais relatados configuram situações para as quais os AINE sejam erroneamente indicados ou representem a expressão de uma reação adversa a estes medicamentos.

Em relação à história prévia de úlcera e/ ou hemorragia digestiva, era de se esperar uma associação negativa entre este fator e o uso de AINE, e este foi um dos resultados da pesquisa. No entanto, vale dizer que Chrischilles et al. (1990) ${ }^{16}$, num estudo de prevalência e características do uso de múltiplos analgésicos entre idosos, evidenciaram que as mulheres idosas com história de úlceras utilizavam concomitantemente salicilatos e outros AINE numa freqüência maior do que aquelas não portadoras de úlceras, apesar do risco aumentado de efeitos adversos gastrintestinais nestes indivíduos. $\mathrm{O}$ fato de $15,7 \%$ dos pacientes do nosso estudo que tinham antecedentes de úlcera e/ou hemorragia utilizarem AINE levanta questões sobre a qualidade deste uso, bem como sobre a existência de monitoramento destes pacientes, o que pode ser objeto de investigações futuras

O estudo da associação entre o uso de corticosteróide e o uso de AINE foi motivado pela interação destes dois fatores no aumento do risco de lesões e complicações de lesões do trato gastrintestinal ${ }^{19}$, a exemplo do que ocorre com a história prévia de úlcera ou hemorragia. No presente estudo, embora a freqüência geral do uso de corticosteróide tenha sido baixa $(3,0 \%)$, entre estes usuários o uso concomitante com AINE foi alto $(75,0 \%)$, ocorrendo o uso conjunto crônico em $47,0 \%$ dos usuários de corticosteróide. É sabido que o uso associado de corticosteróide e AINE ocorre com maior freqüência no tratamento de doenças reumáticas, principalmente a artrite reumatóide. Carvalho \& Xavier (2001) ${ }^{20}$ apontam que na prática médica nacional, o uso de corticosteróides no tratamento da artrite reumatóide tem sido abusivo e mais intenso que o desejável, principalmente porque muitos médicos não estão habituados aos manejos terapêuticos não farmacológicos de que os portadores desta patologia necessitam. A associação evidenciada, na análise bivariada, entre o uso de corticosteróide e o uso de AINE não se manteve na análise multivariada. A baixa freqüência de pacientes que utilizavam corticosteróides ${ }^{16}$ limitou o poder do estudo para análise dessa variável. De qualquer maneira, o fato de o uso concomitante de AINE e corticosteróide ter sido alto pode indicar uma tendência de coprescrição destes dois fármacos no tratamento de doenças reumáticas na prática médica. Esse fato sugere a necessidade de aprofundamento no estudo desta questão, tendo em vista que pouco se conhece sobre o perfil de utilização de anti-reumáticos no Brasil, mas há indícios de uso abusivo de corticosteróides nesse cenário ${ }^{20,21}$.

O uso regular de quatro ou mais medicamentos também foi positiva e significativamente associado ao uso de AINE. Neste sentido, é necessário observar que, se o número de medicamentos utilizados servir como um indicador do estado de saúde da população estudada, nosso achado pode sugerir que este maior uso de medicamentos representa um maior nível de morbidade da população ou ainda a existência de doenças subjacentes crônicas ou mais complicadas que, em algumas situações, aumentam o risco destes indivíduos sofrerem problemas gastrintestinais ${ }^{19}$. Por outro lado, se o número de medicamentos utilizados significar em algumas situações não um estado precário de saúde, mas, acima de tudo, um sobreconsumo de medicamentos, configura-se então mais um cenário extremamente propício para a ocorrência de interações medicamentosas e reações adversas, principais problemas da polifarmácia. Nitidamen- 
te, os AINE estão presentes em grande parte dos estudos abordando a questão da polifarmácia ${ }^{22-24}$. Este fato respalda a associação observada no presente estudo e evidencia um ambiente crítico diante do potencial particular destes fármacos de se associarem à ocorrência de reações adversas e ao seu potencial de interações medicamentosas significativas com outras classes farmacêuticas de uso crônico.

Os resultados mostram que as características dos pacientes que fizeram uso de AINE no período investigado apresentam semelhanças, mas também algumas diferenças, com as observadas em outros estudos. Ressalte-se aqui as dificuldades de comparação, tendo em vista que nossos resultados referem-se a uma amostra particular composta de pacientes submetidos a endoscopia do trato digestivo alto.

Em linhas gerais, os fatores associados ao uso de AINE evidenciados pelo estudo mostram-se pertinentes à discussão apresentada na literatura: o sexo feminino é um importante preditor do uso destes medicamentos em todos os estudos, ao passo que idade, situação sócio-econômica e hábitos de vida mostram-se controversos. A associação observada no estudo entre o uso destes fármacos e a presença de sintomas gastrintestinais é um fato preocupante que merece atenção especial na prática médica e deve ser colocado como objeto de futuras investigações farmacoepidemiológicas.

O uso de fármacos em geral está intimamente relacionado à presença de doenças. No entanto, é importante lembrar que a decisão de utilizar a grande maioria dos medicamentos é complexa e, assim, os profissionais de saúde precisam compreender melhor os diferentes fatores relacionados ao uso de AINE, bem como necessitam dar uma maior atenção aos subgrupos de uso evidenciados pelo estudo.

O presente estudo analisou fatores associados ao uso de AINE. Certamente, os fatores e sua expressão podem diferir ao se considerar diferentes tipos de uso de AINE, diferentes especialidades farmacêuticas e diferentes orientações de uso em diversos grupos populacionais. Percebida a sua relevância e complexidade crítica, faz-se necessário um aprofundamento no estudo desta questão, principalmente no contexto social brasileiro.

\section{Referências}

1. Motola D et al. Pattern of NSAID use in the Italian general population: a questionnaire-based survey. Eur J Clin Pharmacol 2004; 60(10): 731-8.

2. Porteus T, Bond C, Hannaford P, Sinclair H. How and why are non-prescription analgesics used in Scotland? Fam Pract 2005; 22(1): 78-85.

3. Pilotto A, Franceschi M, Leandro G, Di Mario F. NAID and Aspirin use by the elderly in general practice. Drugs Aging 2003; 20(9): 701-10.

4. Paulose-Ram R, Hirsch R, Dillon C, Losonczy K, Cooper $\mathrm{M}$, Ostchega Y. Prescription and non-prescription analgesic use among the US adult population: results from the third National Health and Nutrition Examination Survey (NHANES III).

Pharmacoepidemiol Drug Saf 2003; 12(4): 315-26.

5. Wolfe MM, Lichtenstein DR, Singh G. Gastrointestinal toxicity of nonsteroidal antiinflammatory drugs. $N$ Engl J Med 1999; 340(24): 1888-99.
6. Beria JU, Victora CG, Barros FC, Teixeira AB, Lombardi C. Epidemiologia do consumo de medicamentos em crianças no centro urbano da região sul do Brasil. Rev Saúde Pública 1993; 27(2): 95-104.

7. Loyola Filho AI, Uchoa E, Guerra HL, Firmo JO, LimaCosta MF. Prevalência e fatores associados à automedicação: resultados do Projeto Bambuí. Rev Saúde Pública 2002; 36 (1): 55-62.

8. Noronha V. Aspectos do consumo de medicamentos em uma maternidade de médio porte [monografia]. Belo Horizonte (MG): Universidade Federal de Minas Gerais; 1999.

9. Antonov K, Isacson D. Use of analgesics in Sweden the importance of sociodemographic factors, physical fitness, health and health-related factors, and working conditions. Soc Sci Med 1996; 42(11):1473-81.

10. Baum C, Kennedy DL., Forbes, MB. Utilization of nonsteroidal antiinflammatory drugs. Arthritis Rheum 1985; 28(6): 686-92. 
11. Eggen AE. The Tromso study: frequency and predicting factors of analgesic drug use in a free-living population (12 - 56 years). J Clin Epidemiol 1993; 46 (11): 1297-1304.

12. Ferguson D. Smoking, drinking and non-narcotic analgesic habits in an ocupational group. Med J Aust 1973; 1: 1271-4.

13. Ribeiro AQ. Estudo epidemiológico do uso de antiinflamatórios não-esteroidais por pacientes submetidos à endoscopia no Hospital das Clínicas da UFMG [dissertação de mestrado]. Belo Horizonte: Universidade Federal de Minas Gerais; 2002.

14. Pepe VLE. Estudo sobre a prescrição de medicamentos em uma Unidade de Atenção Primária [dissertação de mestrado]. Rio de Janeiro: Universidade do Estado do Rio de Janeiro; 1994.

15. Isacson D, Bingefors K. Epidemiology of analgesic use: a gender perspective. Eur J Anaes-thesiol Suppl 2002; 26: $5-15$.

16. Chrischilles EA, Lemke JH, Wallace, RB, Drube GA. Prevalence and characteristics of multiple analgesic drug use in an elderly study group. J Am Geriatr Soc 1990; 38: 979-84.

17. Cullen DJ et al. Pharmacoepidemiology of nonsteroidal anti-inflammatory drug use in Nottingham general practices. Aliment Pharmacol Ther 2000; 14(2):177-85.
18. Hogan DB, Campbell NRC, Crutcher R, MC Leod N. Prescription of nonsteroidal anti-inflammatory drugs for elderly people in Alberta. Can Med Assoc J 1994; $151(3): 315-22$.

19. Piper JM, Ray WA, Daugherty JR, Griffin MR. Corticosteroid use and peptic ulcer disease: role of nonsteroidal anti-inflammatory drugs. Ann Intern Med 1991; 114(9): 735-40.

20. Carvalho MAP, Xavier AJD. Artrite reumatóide. In: Moreira C, Carvalho MAP. Reumatologia - diagnóstico e tratamento. 2a.ed. Rio de Janeiro: Medsi; 2001. p. 371-89.

21. Ferraz MB, Pereira RB, Paiva JG, Atra E, dos Santos JQ. Availability of over-the-counter drugs for arthritis in São Paulo, Brazil. Soc Sci Med 1996; 42(8): 1129-31.

22. Bjerrum L, Sogaard J, Hallas J, Kragstrup J. Polypharmacy: correlations with sex, age and drug regimen. Eur J Clin Pharmacol 1998; 54: 197-202.

23. Klaukka T, Makela M, Sipila J, Martikainen J. Multiuse of medicines in Finland. Med Care 1993; 31(5): 445-50.

24. Simons LA et al. Multiple medication use in the elderly: use of prescription and non-prescription drugs in an Australian community setting. Med J Aust 1992; 157(4): 242-6.

recebido em: 18/04/05

versão final reapresentada em: 27/06/05

aprovado em: 30/08/05 